\title{
Expression of MMP-2, MMP-9, MMP-14, TIMP-1, TIMP-2 in Intraepithelial and Invasive Cervical Neoplasia
}

Maria CA Westin¹, Silvia H Rabelo-Santos ${ }^{2}$, Liliana AL Ângelo-Andrade ${ }^{3}$, Sophie Derchain¹, Glauce A Pinto ${ }^{4}$, Sirlei S Morais ${ }^{1}$, Paulo Latuf

Filho $^{4}$ and Luiz Carlos Zeferino ${ }^{1 *}$

${ }^{1}$ Department of Obstetrics and Gynecology, State University of Campinas - UNICAMP, Campinas, Brazil

${ }^{2}$ School of Pharmacy, Federal University of Goiás, UFG, Goiânia, Brazil

${ }^{3}$ Department of Pathology, State University of Campinas - UNICAMP, Brazil

${ }^{4}$ Laboratory of Pathology, CAISM-Prof Dr Jose Aristodemo Pinotti Women's Hospital, State University of Campinas - UNICAMP, Brazil

\begin{abstract}
Aim: To evaluate the expression of MMP-2, MMP-9, MMP-14, TIMP-1 and TIMP-2 in Cervical intraepithelial neoplasia grade $3(\mathrm{CIN} 3)$ and invasive squamous cell carcinoma.

Methods and Results: This study comprised three groups: Group 1: 55 cases with CIN 3; Group 2: 30 cases with CIN 3 and invasive carcinoma components; Group 3: 46 cases with invasive carcinoma. Protein expression was investigated in tumor and stromal cells by immunohistochemistry and percentages of immunostained cells were determined. The mean percentage of MMP-14 tumor cells was significantly higher in stromal cells in all groups. TIMP-2 and MMP-9 expression was significantly higher in stromal cells than in tumor cells. TIMP-1 had a significantly higher expression in stromal cells of carcinoma and tumor cells of CIN 3. Stromal cells expression of MMP-2, MMP14 , TIMP-1 was increased with the severity of cervical neoplasia. The expression of MMP-2 in stromal cells that was higher in CIN 3 component of Group 2 than in CIN 3 of Group 1.
\end{abstract}

Conclusions: The increase in MMP-2 expression from CIN 3 to invasive cervical cancer reinforces their role in cervical cancer progression. From CIN 3 to invasive cervical carcinoma, TIMP-1 expression increased in stromal cells and decreased in tumor cells.

Keywords: Matrix metalloproteinase; Tissue inhibitor of metalloproteinases; Cervical intraepithelial neoplasia; Uterine cervical neoplasms; Neoplasm invasiveness; Carcinogenesis

\section{Introduction}

Cervical intraepithelial neoplasia grade $3(\mathrm{CIN} 3)$ is the precancerous lesion that may progress to invasive carcinoma $[1,2]$. Transformation from preinvasive neoplasia to invasive carcinoma starts by a focal disruption of the subepithelial basement membrane. Potent proteolytic enzymes termed matrix proteinases (MMPs) are known to play a key role in this process [3].

Tumor cells secrete MMPs and induce stromal cells to produce MMPs [4,5]. MMP-2 and MMP-9 are proteolytic enzymes with a major role in type IV collagen digestion, an important event in vascular invasion and metastasis. MMP-14 is a key enzyme in tumor cell invasion. This enzyme seems to be implicated in both breaching the basement membrane by tumor cells and cell invasion through interstitial type-I collagen tissues [6].

Tissue inhibitors of matrix metalloproteinases (TIMPs 1 to 4 ) are natural inhibitors of MMPs. TIMPs are multifunctional proteins that not only inhibit MMPs but also promote cell growth, induce apoptosis, and inhibit angiogenesis [5,7]. However evidence suggests that TIMPs, in particular TIMP-1 and TIMP-2, may have unique biological properties, independent of their ability to inhibit MMPs [8].

Expression of MMP and TIMP markers has been studied mostly in tumor cells. In stromal cells, expression of these markers has been less frequently evaluated. It has been shown that cell-cell contact between cervical carcinoma cells and peripheral stromal fibroblasts augments the production and activation of MMPs. Therefore, the subsequent imbalance between MMPs and TIMPs may result in the gradually increasing invasive potential of cervical cancer cells [9-11].
A better understanding of the mechanisms by which CIN 3 acquires the ability to invade the cervical stroma may have a significant clinical relevance. Therefore, the aim of this study was to describe and analyze the expression of MMP-2, MMP-9, MMP-14, TIMP-1 and TIMP-2 in CIN 3 and invasive carcinoma of the cervix, in tumor cells and stromal cells.

\section{Materials and Methods}

\section{Ethics statement}

All tissue samples were collected for histologic examination and diagnostic purposes and were thoroughly anonymized for the use in this study. This study was approved by the State University of Campinas ethics committee (Approval No. 858/2007 - November 11th, 2007).

\section{Tissue samples}

This comparative observational study of women with cervical neoplasia consisted of three groups. The first group comprised 55 cases of histologic diagnosis of unique CIN 3 (Group 1), the second group comprised 30 cases with both components: CIN 3 (Group 2) and invasive carcinoma (Group 2) and the third group was composed of

*Corresponding author: Luiz Carlos Zeferino,Department of Obstetrics and Gynecology, State University of Campinas - UNICAMP, Campinas, Brazil, Tel: +5519-35219305; Fax: +55-19-35219424; E-mail: zeferino@fcm.unicamp.brr

Received August 28, 2015; Accepted October 19, 2015; Published October 21, 2015

Citation: Westin MC, Rabelo-Santos SH, Ângelo-Andrade LA, Derchain S Pinto GA, et al. (2015) Expression of MMP-2, MMP-9, MMP-14, TIMP-1, TIMP2 in Intraepithelial and Invasive Cervical Neoplasia. J Cytol Histol S3:019. doi:10.4172/2157-7099.S3-019

Copyright: (c) 2015 Westin MC, et al. This is an open-access article distributed under the terms of the Creative Commons Attribution License, which permits unrestricted use, distribution, and reproduction in any medium, provided the original author and source are credited. 
Citation: Westin MC, Rabelo-Santos SH, Ângelo-Andrade LA, Derchain S, Pinto GA, et al. (2015) Expression of MMP-2, MMP-9, MMP-14, TIMP-1, TIMP-2 in Intraepithelial and Invasive Cervical Neoplasia. J Cytol Histol S3:019. doi:10.4172/2157-7099.S3-019

46 cases with a histologic diagnosis of invasive carcinoma (Group 3). The study design was constructed assuming that pure CIN 3 (Group 1), CIN 3 associated with invasive carcinoma (Group 2) and pure cervical invasive carcinoma (Group 3) represent progressive steps from CIN 3 to invasive carcinoma.

Case selection was made from records of pathology reports obtained from women consecutively managed in the Women's Hospital, State University of Campinas, Brazil, between 2002 and 2008. Hematoxilin-eosin paraffin-embedded sections were reviewed and the best representative sample was identified considering tumor tissue and the underlying stroma. Specimens were obtained by punch biopsies, conizations or hysterectomies. The number of cases varied in each analysis, in some cases lesions were not represented for all markers, due to serial section of the tissue block. Despite some limitations of immunohistochemistry analysis of the stroma cells, this tissue compartment was analyzed because there is evidence that the stroma has a relevant role in cervical cancer progression [9].

\section{Immunohistochemistry assay}

Expressions of MMP-2, MMP-9, MMP-14, TIMP-1 and TIMP-2 were investigated in paraffin-embedded sections using the avidinbiotinperoxidase complex method. The paraffin sections ( $5 \mu \mathrm{m}$ thick) were deparaffinized and immersed in 3\% hydrogen peroxidase in methanol to block endogenous peroxidase activity. Next, an antigen retrieval procedure was performed by immersing the slides in $10 \mathrm{mM}$ citrate buffer (pH 6.0) and autoclaving at $121^{\circ} \mathrm{C}$ for $10 \mathrm{~min}$. After washing in PBS, the tissue sections were preblocked using $10 \%$ normal goat serum for $15 \mathrm{~min}$. The protocol for the Dako LSAB 2 peroxidase kit (Dako, Kyoto, Japan) was followed. The sections were incubated overnight with primary antibodies in a humidity chamber at $4^{\circ} \mathrm{C}$.

The primary antibodies used were polyclonal mouse antihuman MMP-2, MMP-9, MMP-14, TIMP-1 and TIMP-2 (Nemarkers). The working dilutions for each primary antibody were: MMP-2 (1:100), MMP-9 (1:200), MMP-14 (1:100), TIMP-1(1:100) and TIMP-2 (1:100). Sections were rinsed with PBS for $15 \mathrm{~min}$ and incubated for $1 \mathrm{~h}$ with the secondary antibody (biotinylated goat anti-mouse and rabbit immunoglobulin G secondary antibody; Dako). The sections were then incubated with streptavidin-peroxidase complex using 3,3'diaminobenzidine as a chromogen. The sections were counterstained with Mayer's hematoxylin. Specificity of the immunohistochemical reactions was checked by omitting the primary antibody.

\section{Immunohistochemistry assay and image acquisition}

The methodology for interpretation of the immunostaining sections consisted in the following steps:

1 - Identification of representative regions of the lesions selected, according to group (CIN 3, invasive carcinoma and underlying stromal lesion); selection criteria for these representative areas were based on: image sharpness, areas of higher intensity of cellular immunoreaction (but any intensity of immunostaining was considered positive), a similar proportion of stromal and tumor region in the same picture, excluding necrotic regions. Stromal regions with a high concentration of inflammatory cells were excluded.

2 - Images of these regions were captured under a magnification of 400X. In Group 2, which included cases of invasive cell carcinoma associated with CIN 3 , pictures were taken separately.
3 - Selection of one photograph per lesion and its underlying stroma was made for quantitative and qualitative analysis.

4 - Calculation of total cell number and number of immunostained cells (stromal and tumor cells separately) was made by two observers using morphometric software (Image Pro Plus ${ }^{\oplus}$, version 6.3, Olympus). To determine the percentage of immunostained cells, at least 1,000 tumor cells and stromal cells per case were counted (Figures 1-3).

\section{Statistical analysis}

Statistical analysis was carried out, considering the mean percentage of immunopositive tumor cells and stromal cells within each group and between diagnostic groups. The CIN 3 and invasive carcinoma components of Group 2 were analyzed separately. The

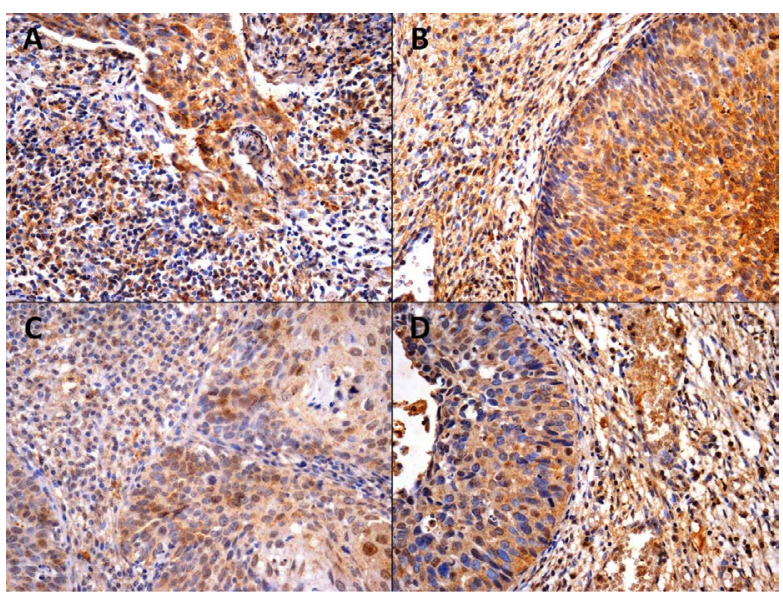

Figure 1: Percentage of tumor and stromal cells expressing MMP-2. A: Invasive carcinoma (Group 3): Stromal cells 97.5\%, Tumor Cells 97.8\%; B: CIN 3 (Group 1): Stromal cells $89.6 \%$, Tumor Cells $100 \%$; C: Invasive carcinoma compartment (Group 2): Stromal cells-88.2\%, Tumor Cells-82.4\%; D: CIN 3 compartment (Group 2): Stromal cells-83.8\%, Tumor Cells-84.5\%. MMP-Metalloproteinases, Cervical Intraepithelial Neoplasia

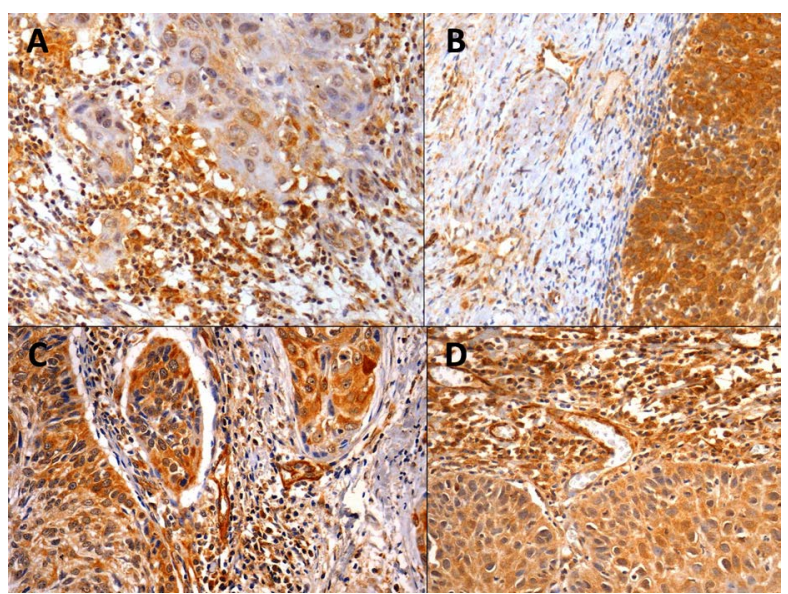

Figure 2: Percentage of tumor and stromal cells expressing TIM- 1. A: Invasive carcinoma (Group 3): Stromal cells $89.6 \%$, Tumor Cells 56,2\%; B: CIN 3 (Group 1): Stromal cells 98.7\%, Tumor Cells-19.8\%; C: Invasive carcinoma compartment (Group 2): Stromal cells-87.2\%, Tumor Cells-98.2\%; D: CIN 3 compartment (Group 2): Stromal cells-88.8\%, Tumor Cells-82.0\%. TIMP-Tissue inhibitors of metalloproteinases, Cervical Intraepithelial Neoplasia 
Citation: Westin MC, Rabelo-Santos SH, Ângelo-Andrade LA, Derchain S, Pinto GA, et al. (2015) Expression of MMP-2, MMP-9, MMP-14, TIMP-1, TIMP-2 in Intraepithelial and Invasive Cervical Neoplasia. J Cytol Histol S3:019. doi:10.4172/2157-7099.S3-019

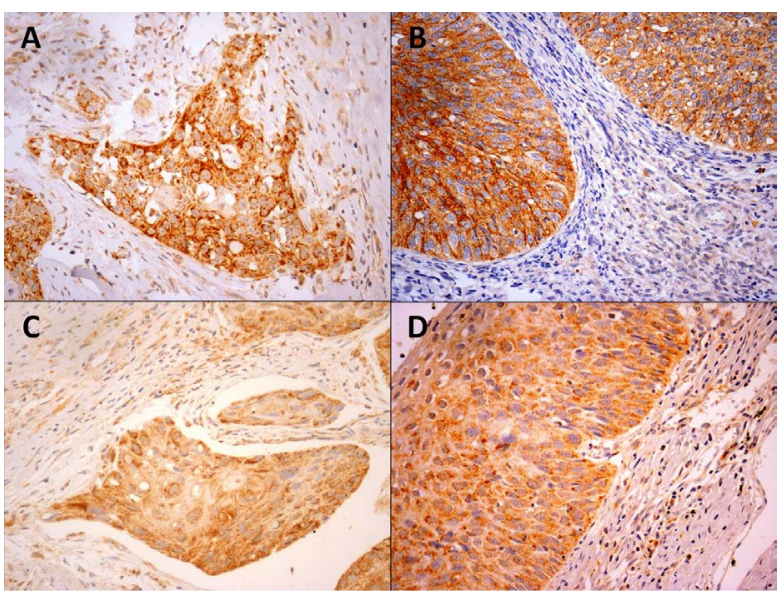

Figure 3: Percentage of tumor and stromal cells expressing MMP14. A: Invasive carcinoma (Group 3): Stromal cells 67\%, Tumor Cells 100\%; B: CIN 3 (Group 1): Stromal cells 21.3\%, Tumor Cells-100\%; C: Invasive carcinoma compartment (Group 2): Stromal cells-62.2\%, Tumor Cells-100\%; D: CIN 3 compartment (Group 2): Stromal cells-49.9\%, Tumor Cells- $100 \%$

MMP-Metalloproteinases, CIN- Cervical Intraepithelial Neoplasia

T-paired test was used to compare the mean percentage of tumor cells and stromal cells within each diagnostic group. The percentages of tumor and stromal cells between two diagnostic groups were analyzed by the Mann-Whitney and Wilcoxon Signed Rank tests. Differences were considered significant when $p$ value was less than 0.05 .

\section{Results}

The MMP-14, MMP-2 and TIMP-1 proteins were markedly expressed in tumor cells and stromal cells most cases.

\section{MMP-14}

In all groups, the mean percentage of cells expressing MMP-14 was significantly higher in tumor than in stromal cells. For tumor cells, the mean percentage ranged from $80.2 \%$ (Group 2 - invasive carcinoma) to $89.1 \%$ (Group 2 - CIN 3); for stromal cells, the mean percentage ranged from $51.9 \%$ (Group 1 - CIN 3) to $66.1 \%$ (Group 3 - invasive carcinoma). Intergroup analysis of tumor cells showed that MMP-14 expression did not vary. Nevertheless, the percentage of stromal cells positive for MMP-14 was significantly higher $(\mathrm{p}=0.0212)$ in invasive carcinoma (Group 3) (median=69.8\%) than in CIN 3 (Group 1) (median=57.2\%) (Tables 1-4 and Figure 4 ).

\section{MMP-2}

In all diagnostic groups, there was no statistically significant difference in the mean percentage of cells expressing MMP-2 in both tumor and stromal cells. The percentages of both tumor and stromal cells immunopositive for MMP-2 were significantly higher ( $\mathrm{p}=0.0066$ and $\mathrm{p}=0.0014$, respectively) in invasive carcinoma (Group 3) compared to CIN 3 (Group 1). The percentage of stromal cells immunopositive for MMP-2 was significantly higher $(\mathrm{p}=0.0141)$ in the CIN 3 component (Group 2) than in CIN 3 (Group 1) (Tables 1-4 and Figure 4).

\section{MMP-9}

The mean percentage of cells expressing MMP-9 was significantly higher in stromal than in tumor cells in the three groups, i.e.: CIN 3 (Group 1), CIN 3 component (Group 2) and invasive carcinoma (Group $3)$. For stromal cells, the mean percentage was $58.8 \%(\mathrm{p}=0.0295)$ in
CIN 3 (Group 1), 65.9\% ( $\mathrm{p}=0.0357)$ in the CIN 3 component (Group 2 ), and $53.2 \%(\mathrm{p}=0.0009)$ in invasive carcinoma (Group 3). For tumor cells, the mean percentage was $49.9 \%, 52.3 \%$ and $42.0 \%$, respectively. When tumor and stromal cells were analyzed, MMP-9 expression did not vary between groups (Table 1-4 and Figure 4).

\section{TIMP-1}

In groups at opposite ends (of the range of severity), the mean percentage of cells expressing TIMP-1 was statistically higher in tumor cells (mean $=83.8 \% ; \mathrm{p}=0.0002$ ) from CIN 3 (Group 1). Inversely, it was statistically higher in stromal cells (mean $=83.7 \% ; \mathrm{p}=0.0076$ ) from invasive carcinoma (Group 3). There was a significantly higher percentage of tumor cells expressing TIMP-1 $(\mathrm{p}=0.0119)$ than stromal cells, when CIN 3 (Group 1) was compared to invasive carcinoma (Group 3). Inversely, there was a significantly higher percentage of stromal cells expressing TIMP-1 $(\mathrm{p}=0.0082)$ than tumor cells, when invasive carcinoma (Group 3) was compared to CIN 3 (Group 1) (Tables 1-4 and Figure 4).

\section{TIMP-2}

In all groups, the mean percentage of cells expressing TIMP-2 was significantly higher in stromal than in tumor cells. In stromal cells, the mean percentage ranged from $59.0 \%(\mathrm{p}<0.0001)$ (CIN 3- Group 1) to $65.1 \%(\mathrm{p}<0.0001)$ (invasive carcinoma - Group 3). In tumor cells, the mean percentage ranged from $37.7 \%$ (invasive carcinoma - Group 3) to $51.1 \%$ (CIN 3 component - Group 2).. However, TIMP-2 expression in tumor or stromal cells showed no statistical difference between the diagnostic groups examined (Tables 1-4 and Figure 4).

\begin{tabular}{|c|c|c|c|c|c|c|c|c|c|c|}
\hline Markers & Cells & $\mathrm{n}$ & Mean & SD & Min & Q1 & Median & Q3 & Max & $p$-value \\
\hline \multirow{2}{*}{ MMP2 } & Tumor & 51 & 58.42 & 26.42 & 11.66 & 38.82 & 50.64 & 82.33 & 100.0 & \multirow{2}{*}{0.3245} \\
\hline & Stromal & 51 & 55.32 & 21.48 & 21.44 & 34.31 & 52.78 & 71.31 & 99.15 & \\
\hline \multirow{2}{*}{ MMP9 } & Tumor & 55 & 49.87 & 29.85 & 1.62 & 26.14 & 47.71 & 77.65 & 99.65 & \multirow{2}{*}{0.0295} \\
\hline & Stromal & 55 & 58.78 & 29.58 & 0.62 & 41.0 & 62.49 & 85.15 & 97.98 & \\
\hline \multirow{2}{*}{ MMP14 } & Tumor & 51 & 86.25 & 20.41 & 8.43 & 78.91 & 97.84 & 100.0 & 100.0 & \multirow{2}{*}{$\begin{array}{c}< \\
0.0001\end{array}$} \\
\hline & Stromal & 51 & 51.89 & 28.53 & 1.25 & 28.8 & 57.17 & 75.68 & 99.79 & \\
\hline \multirow{2}{*}{ TIMP1 } & Tumor & 53 & 83.75 & 14.81 & 42.49 & 77.1 & 84.29 & 96.98 & 100.0 & \multirow{2}{*}{0.0002} \\
\hline & Stromal & 53 & 72.31 & 20.3 & 19.77 & 56.64 & 71.67 & 88.46 & 100.0 & \\
\hline \multirow{2}{*}{ TIMP2 } & Tumor & 52 & 42.08 & 25.73 & 0.77 & 23.29 & 37.53 & 56.22 & 96.39 & \multirow{2}{*}{$\begin{array}{c}< \\
0.0001\end{array}$} \\
\hline & Stromal & 52 & 59.04 & 25.58 & 5.72 & 35.0 & 67.77 & 80.17 & 94.34 & \\
\hline & r. & & H & 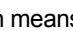 & or & ffr & 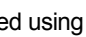 & $n$ & $d t$ & \\
\hline
\end{tabular}

Table 1: Comparison of mean percentages of tumor and stromal cells immunopositive in CIN 3 (Group 1).

\begin{tabular}{|c|c|c|c|c|c|c|c|c|c|c|}
\hline Markers & Cells & $\mathrm{n}$ & Mean & SD & Min & Q1 & Median & Q3 & Max & $p$-value \\
\hline \multirow{2}{*}{ MMP2 } & Tumor & 30 & 63.81 & 30.13 & 4.9 & 38.5 & 75.46 & 90.48 & 99.36 & \multirow{2}{*}{0.5244} \\
\hline & Stromal & 30 & 67.8 & 26.15 & 6.9 & 51.54 & 77.86 & 88.3 & 98.2 & \\
\hline \multirow{2}{*}{ MMP9 } & Tumor & 26 & 52.28 & 32.32 & 1.73 & 15.87 & 54.03 & 84.57 & 94.29 & \multirow{2}{*}{0.0357} \\
\hline & Stromal & 26 & 65.94 & 24.13 & 9.77 & 43.35 & 74.17 & 85.23 & 93.56 & \\
\hline \multirow{2}{*}{ MMP14 } & Tumor & 27 & 89.13 & 17.48 & 35.79 & 84.17 & 100.0 & 100.0 & 100.0 & \multirow{2}{*}{$\begin{array}{c}< \\
0.0001\end{array}$} \\
\hline & Stromal & 27 & 56.46 & 24.95 & 11.39 & 40.29 & 56.93 & 81.46 & 92.67 & \\
\hline \multirow{2}{*}{ TIMP1 } & Tumor & 18 & 81.58 & 16.73 & 45.74 & 67.3 & 86.73 & 95.78 & 99.79 & \multirow{2}{*}{0.7674} \\
\hline & Stromal & 18 & 82.81 & 15.62 & 48.1 & 71.84 & 84.82 & 96.37 & 100.0 & \\
\hline \multirow{2}{*}{ TIMP2 } & Tumor & 19 & 51.11 & 23.96 & 5.19 & 27.27 & 60.97 & 68.59 & 79.48 & \multirow{2}{*}{0.0123} \\
\hline & Stromal & 19 & 63.91 & 22.04 & 18.39 & 42.73 & 64.24 & 79.18 & 96.46 & \\
\hline & & & & & & & & & & \\
\hline
\end{tabular}

Table 2: Comparison of mean percentages of tumor and stromal cells immunopositive in CIN 3 (Group 2). 
Citation: Westin MC, Rabelo-Santos SH, Ângelo-Andrade LA, Derchain S, Pinto GA, et al. (2015) Expression of MMP-2, MMP-9, MMP-14, TIMP-1, TIMP-2 in Intraepithelial and Invasive Cervical Neoplasia. J Cytol Histol S3:019. doi:10.4172/2157-7099.S3-019

\begin{tabular}{|l|c|c|c|c|c|c|c|c|c|c|}
\hline Markers & Cells & $\mathrm{n}$ & Mean & SD & Min & Q1 & Median & Q3 & Max & p-value \\
\hline \multirow{2}{*}{ MMP2 } & Tumor & 30 & 64.85 & 26.87 & 12.1 & 45.63 & 72.71 & 85.99 & 99.27 & \multirow{2}{*}{0.7785} \\
\cline { 2 - 11 } & Stromal & 30 & 66.54 & 20.39 & 13.57 & 55.08 & 67.81 & 81.35 & 100.0 & \\
\hline \multirow{2}{*}{ MMP9 } & Tumor & 26 & 54.68 & 31.59 & 0.71 & 28.61 & 55.49 & 85.62 & 100.0 & \multirow{2}{*}{0.2659} \\
\cline { 2 - 11 } & Stromal & 26 & 63.19 & 28.87 & 0.17 & 35.37 & 75.36 & 86.33 & 100.0 & \\
\hline \multirow{2}{*}{ MMP14 } & Tumor & 27 & 80.18 & 26.26 & 17.21 & 54.27 & 100.0 & 100.0 & 100.0 & \multirow{2}{*}{0.0224} \\
\cline { 2 - 11 } & Stromal & 27 & 64.96 & 27.93 & 14.58 & 45.3 & 74.57 & 87.32 & 98.14 & \\
\hline \multirow{2}{*}{ TIMP1 } & Tumor & 18 & 82.3 & 15.12 & 54.07 & 74.85 & 88.41 & 93.32 & 99.51 & \multirow{2}{*}{0.9072} \\
\cline { 2 - 10 } & Stromal & 18 & 82.76 & 16.03 & 33.94 & 76.74 & 86.15 & 94.26 & 98.96 & \\
\hline \multirow{2}{*}{ TIMP2 } & Tumor & 19 & 44.3 & 19.65 & 14.69 & 28.59 & 42.47 & 53.01 & 78.76 & \multirow{2}{*}{0.0174} \\
\cline { 2 - 6 } & Stromal & 19 & 61.15 & 16.9 & 8.43 & 55.24 & 61.9 & 71.33 & 89.4 & \\
\hline
\end{tabular}

Comparisons between means were performed using the paired t-test

Table 3: Comparison of mean percentages of tumor and stromal cells immunopositive in Invasive Carcinoma (Group 2)

\begin{tabular}{|c|c|c|c|c|c|c|c|c|c|c|}
\hline Markers & Cells & $\mathrm{n}$ & Mean & SD & Min & Q1 & Median & Q3 & Max & p-value \\
\hline \multirow{2}{*}{ MMP2 } & Tumor & 37 & 76.05 & 20.14 & 28.58 & 64.81 & 82.99 & 91.39 & 99.96 & \multirow{2}{*}{0.1953} \\
\cline { 2 - 10 } & Stromal & 37 & 69.99 & 18.87 & 6.51 & 62.21 & 72.99 & 84.9 & 97.81 & \\
\hline \multirow{2}{*}{ MMP9 } & Tumor & 46 & 42.01 & 38.56 & 0.07 & 3.45 & 35.74 & 81.42 & 100.0 & \multirow{2}{*}{0.0009} \\
\cline { 2 - 10 } & Stromal & 46 & 53.2 & 35.51 & 0.07 & 17.89 & 66.23 & 85.74 & 95.41 & \\
\hline \multirow{2}{*}{ MMP14 } & Tumor & 35 & 85.75 & 25.15 & 3.39 & 83.23 & 100.0 & 100.0 & 100.0 & $<$ \\
\cline { 2 - 9 } & Stromal & 35 & 66.06 & 23.79 & 10.9 & 45.64 & 69.83 & 87.49 & 100.0 & 0.0001 \\
\hline \multirow{2}{*}{ TIMP1 } & Tumor & 46 & 76.53 & 15.08 & 40.26 & 68.03 & 77.66 & 88.45 & 99.65 & \multirow{2}{*}{0.0076} \\
\cline { 2 - 9 } & Stromal & 46 & 83.72 & 11.64 & 59.55 & 76.37 & 84.52 & 93.81 & 100.0 & \\
\hline \multirow{2}{*}{ TIMP2 } & Tumor & 38 & 37.69 & 24.58 & 0.0 & 17.42 & 37.44 & 49.46 & 88.57 & \multirow{2}{*}{$<$} \\
\cline { 2 - 7 } & Stromal & 38 & 65.11 & 21.73 & 3.13 & 49.08 & 64.21 & 86.38 & 100.0 & 0.0001 \\
\hline \multirow{2}{*}{ Comparisons between means were performed using the paired t-test }
\end{tabular}

Table 4: Comparison of mean percentages of tumor and stromal cells immunopositive in Invasive Carcinoma (Group 3)

\section{Discussion}

The analyzed markers were similarly or more highly expressed in stromal cells than in tumor cells, except for MMP-14 expression, that was more expressed in tumor cell. In all groups, MMP-2 expression was similar in tumor and stromal cells. The marker TIMP-1 was the unique that changed the distribution between tumor and stromal cells, since TIMP-1 expression was higher in tumor cells of CIN 3, and higher in stromal cells of invasive carcinoma.

Similarly to this study, Nair et al. [10] observed that MMP-2, MMP-9 and TIMPs showed intense immunostaining in both stromal and tumor cells of invasive carcinoma in virtually all cases. Neoplastic changes occur in epithelial cells, but there is also a significant change in MMP and TIMP expression in stromal cells, reinforcing that stromal cells play an important role in the carcinogenic process. Under normal physiological conditions, the stroma acts as an important barrier to epithelial cell transformation. Nevertheless, the stromal compartment undergoes changes in response to emerging epithelial lesions. This compartment has a key role in cancer initiation and progression, including the recruitment of new stromal cells that provide factors involved in cell growth and matrix remodeling $[11,12]$.

Although this study showed that MMP-2, MMP-14 and TIMP1 were highly expressed in all groups, major differences in marker expression were found between CIN 3 (Group 1) and invasive carcinoma (Group 3). MMP-2 expression was higher in tumor and stromal cells of invasive carcinoma than in these cells of CIN 3. This marker also showed higher expression in stromal cells of CIN 3 of Group 2 than in stromal cells of CIN 3 of Group 1. MMP-14 was more expressed in stromal cells of invasive carcinoma than in stromal cells of CIN 3. There was a different trend in TIMP-1 expression from CIN
3 to invasive carcinoma in tumor and stromal cells. TIMP-1 expression was higher in tumor cells of CIN 3 and, inversely, was higher in stromal cells of invasive carcinoma. There was a similar expression of MMP-9 and TIMP-2 in invasive carcinoma and CIN 3.

Although MMP-2 and MMP-9 are almost identical proteinases, their contribution to biological or pathological processes can be very different. At present, it is still unclear which of these enzymes is more important in tumor progression and metastasis [13]. Evidence shows that MMP-2 is especially important in ECM degradation, cancer cell invasion and metastasis. Therefore, MMP-2 is a determinant of cancer cell behavior $[10,13,14]$. This study demonstrated a higher MMP-2 expression in tumor and stromal cells of invasive cervical carcinoma than in the corresponding cells of CIN 3. In addition, MMP-2 was statistically more expressed in CIN 3 stromal cells of Group 2.
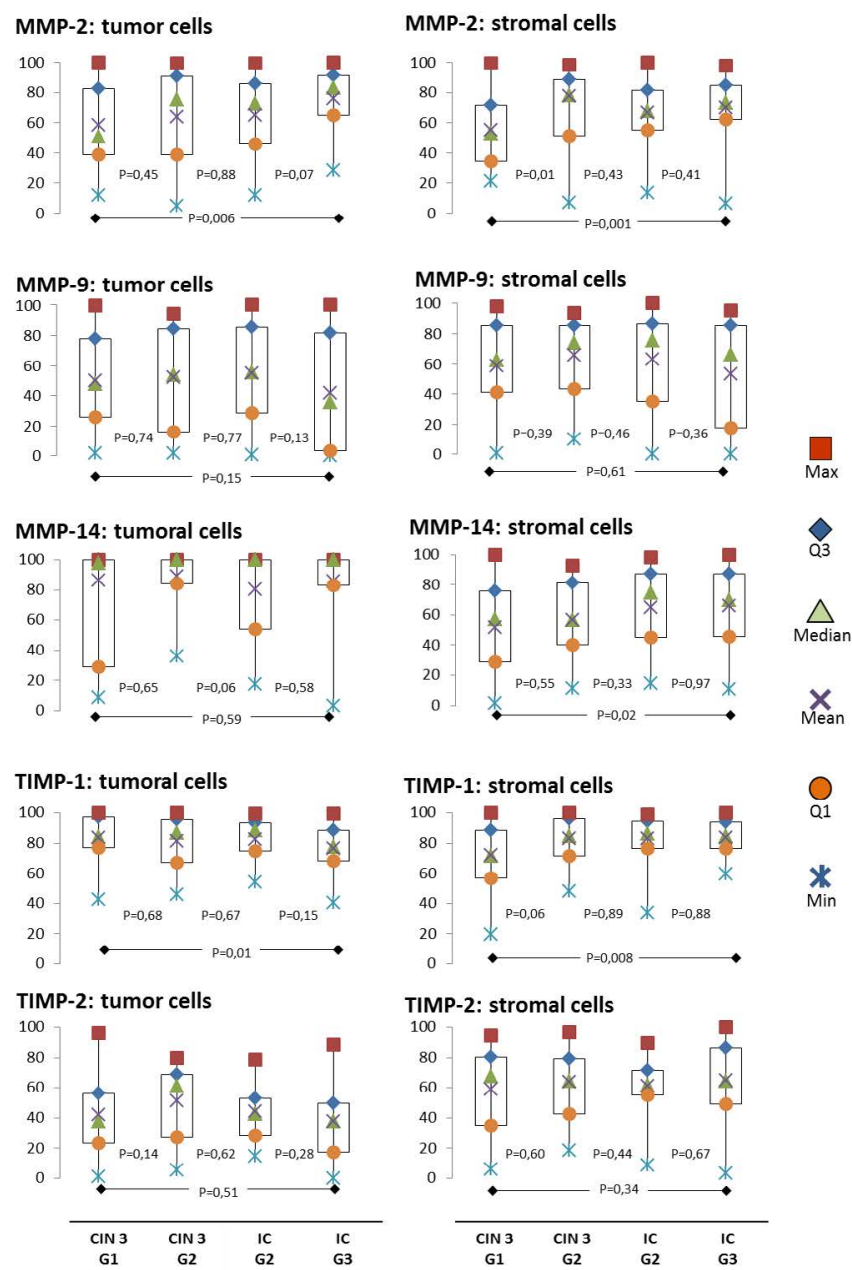

Figure 4: MMP-2, MMP 9, MMP 14, TIM-1 and TIMP 2 expressions in Cervical Neoplasia MMP- Metalloproteinases, TIMP- Tissue inhibitors of metalloproteinases, CIN - Cervical Intraepithelial Neoplasia, IC - Invasive carcinoma, Group 1-G1, Group-G2, Group 3-G3, Min-Minimum, MaxMaximum, Q1- 25th percentile, Q3-75th percentile.

Higher percentages of stromal and tumoral cells expressing MMP 2 were observed in invasive carcinoma (Group 3). Higher percentages of stromal cells expressing MMP-2 were detected in CIN 3 of Group 2 when compared with CIN 3 of Group 1 . There was a significantly higher percentage of tumor cells expressing TIMP-1 than stromal cells, when CIN 3 of Group 1 was compared to invasive carcinoma of Group 3. There was a significantly higher percentage of stromal cells expressing TIMP-1 than tumor cells, when invasive carcinoma of Group 3 was compared to CIN 3 of Group 1. 
Citation: Westin MC, Rabelo-Santos SH, Ângelo-Andrade LA, Derchain S, Pinto GA, et al. (2015) Expression of MMP-2, MMP-9, MMP-14, TIMP-1, TIMP-2 in Intraepithelial and Invasive Cervical Neoplasia. J Cytol Histol S3:019. doi:10.4172/2157-7099.S3-019

However, no difference was demonstrated when the expression of this protein in tumor and stromal cells within the same group was analyzed. In contrast, no difference in MMP-9 expression was demonstrated between CIN 3 and invasive carcinoma, however, this marker showed a different pattern of expression, which was higher in stromal than in tumor cells in almost all groups. These findings suggest that MMP-2 could have a role in the neoplastic progression of the lesion and MMP9 would act through an interaction between tumor and stromal cells.

In agreement with results of this study, Nair et al. [10] also found a progressive increase in MMP-2 positivity related to the severity of cervical neoplasia. Fernandes et al. [15] also showed that MMP-2 expression was higher in stromal cells of invasive carcinomas than of CIN 3 and indicated that stromal cells play an important role in tumor invasion and progression, mediated by the progressive enhancement of MMP-2 expression from CIN 3 to advanced invasive tumor. Brummer et al. [16] suggested that MMP-2 expression, when focally observed in high-grade intraepithelial lesions of the cervix, may indicate tumor regions with an increased risk for invasive growth.

MMP-14 may have a very important role in tumor cell progression. Cell invasion is a multistep process, involving degradation of the extracellular matrix (ECM) and cell mobility. While ECM degradation can be orchestrated collectively by MMPs, cell migration is likely to be predominantly associated with MMP-14 [17]. This understanding is considered concordant with findings in this study which showed that MMP-14 expression was higher in stromal cells in invasive carcinoma than in stromal cells of CIN 3.

Recent studies have demonstrated that TIMP-1 possesses additional functions, including enhancement of malignant transformation, stimulation of cell growth and inhibition of apoptosis, as well as promotion of migration, invasion and angiogenesis. This indicates a potential tumor-promoting role of TIMP-1 in the early stages of tumorigenesis [18]. Elevated TIMP-levels have been reported in association with cancer progression and were identified as poor prognostic indicators in several human types of tumor. This study showed a higher TIMP-1 expression in CIN 3 tumor cells, where malignant transformation, stimulation of cell growth and inhibition of apoptosis occur. In invasive carcinoma, this study showed a higher TIMP-1 expression in stromal cells, where the promotion of migration, invasion and angiogenesis take place. While MMPs are important in the late stage of tumor progression leading to metastasis, the anti-apoptotic effects of some TIMPs may favor tumor growth during tumor onset and early primary tumor growth [19].

The power of this study was the design that analyzed three steps of cervical cancer progression ranging from CIN 3 to invasive carcinoma. However, findings would be more conclusive, if normal cervical tissue had been included as control group. Limitations of the study were subjectivity and variability in immunostaining evaluation.

\section{Conclusions}

The progression of CIN 3 to invasive carcinoma seems be determined by complex interactions between tumor and stromal cells. These findings reinforce the evidence of the role of stromal cells in tumor invasiveness.

\section{Acknowledgements}

This study was supported by FAPESP grant number: 2007/54709-0

\section{References}

1. http://globocan.iarc.fr/
2. Snijders PJ, Steenbergen RD, Heideman DA, Meijer CJ (2006) HPV-mediated cervical carcinogenesis: concepts and clinical implications. J Pathol 208: 152-164.

3. Wu YC, Wang PH, Tsai A, Yang SF, Chen SC (2011) Semi-quantitative expression of tissue inhibitor of matrix metalloproteinase-2 in cancer of uterine cervix. J Surg Oncol 104: 210-215.

4. Yoon SO, Park SJ, Yun CH, Chung AS (2003) Roles of matrix metalloproteinases in tumor metastasis and angiogenesis. J Biochem Mol Biol 36: 128-137.

5. Boyd S, Tolvanen K, Virolainen S, Kuivanen T, Kyllönen L, et al. (2008) Differential expression of stromal MMP-, MMP-9 and TIMP-1 in basal cell carcinomas of immunosuppressed patients and controls. Virchows Arch 452: 83-90.

6. Poincloux R, Lizárraga F, Chavrier $P$ (2009) Matrix invasion by tumour cells a focus on MT1-MMP trafficking to invadopodia. J Cell Sci 122: 3015-3024.

7. Brew K, Dinakarpandian D, Nagase H (2000) Tissue inhibitors of metalloproteinases: evolution, structure and function. Biochim Biophys Acta 1477: 267-283.

8. Stetler-Stevenson WG (2008) The tumor microenvironment: regulation by MMP-independent effects of tissue inhibitor of metalloproteinases-2. Cancer Metastasis Rev 27: 57-66.

9. Sato T, Sakai T, Noguchi Y, Takita M, Hirakawa S, et al. (2004) Tumor-stromal cell contact promotes invasion of human uterine cervical carcinoma cells by augmenting the expression and activation of stromal matrix metalloproteinases. Gynecol Oncol 92: 47-56.

10. Asha Nair S, Karunagaran D, Nair MB, Sudhakaran PR (2003) Changes in matrix metalloproteinases and their endogenous inhibitors during tumor progression in the uterine cervix. J Cancer Res Clin Oncol 129: 123-131.

11. TIsty TD, Coussens LM (2006) Tumor stroma and regulation of cancer development. Annu Rev Pathol 1: 119-150.

12. Del Casar JM, González LO, Alvarez E, Junquera S, Marín L, et al. (2009). Comparative analysis and clinical value of the expression of metalloproteinases and their inhibitors by intratumor stromal fibroblasts and those at the invasive front of breast carcinomas. Breast Cancer Res Treat 116: 39-52.

13. Zhang X, Wang Y, Yamamoto G, Tachikawa T (2009). Expression of matrix metalloproteinases MMP-2, MMP-9 and their tissue inhibitors TIMP-1 and TIMP-2 in the epithelium and stroma of salivary gland pleomorphic adenomas. Histopathology 55: 250-260.

14. Xu X, Wang Y, Chen Z, Sternlicht MD, Hidalgo M, et al. (2005) Matrix metalloproteinase-2 contributes to cancer cell migration on collagen. Cancer Res 65: 130-136.

15. Fernandes T, de Angelo-Andrade LA, Morais SS, Pinto GA, Chagas CA, et al. (2008) Stromal cells play a role in cervical cancer progression mediated by MMP-2 protein. Eur J Gynaecol Oncol 29: 341-344.

16. Brummer O, Böhmer G, Hollwitz B, Flemming P, Petry KU, et al. (2002) MMP-1 and MMP-2 in the cervix uteri in different steps of malignant transformation--an immunohistochemical study. Gynecol Oncol 84: 222-227.

17. Sbardella D, Fasciglione GF, Gioia M, Ciaccio C, Tundo GR, et al. (2012) Human matrix metalloproteinases: an ubiquitarian class of enzymes involved in several pathological processes. Mol Aspects Med 33: 119-208.

18. Jensen SA, Vainer B, Bartels A (2010) Expression of matrix metalloproteinase 9 (MMP-9) and tissue inhibitor of metalloproteinases 1 (TIMP-1) by colorectal cancer cells and adjacent stroma cells - Associations with histopathology and patients outcome. European J Cancer 46: 3233-3242.

19. Jiang Y, Goldberg ID, Shi YE (2002) Complex roles of tissue inhibitors of metalloproteinases in cancer. Oncogene 21: 2245-2252.

This article was originally published in a special issue, Cyłopathology handled by Editor(s). Borislav A. Alexiev. Department of Pathology University of Maryland Medical Center, USA 